\title{
Lymphocytic Colitis in Nigeria: A Case Series
}

\author{
Aderemi Oluyemi ${ }^{1}$, Martins Momoh ${ }^{2}$, Emuobor Odeghe ${ }^{3}$, Samuel Keshinro ${ }^{4}$ \\ ${ }^{1}$ ReMay Consultancy \& Medical Services, Ikeja, Lagos, Nigeria \\ ${ }^{2}$ Department of Morbid Anatomy, Lagos University Teaching Hospital, Lagos, Nigeria \\ ${ }^{3}$ Department of Medicine, College of Medicine, University of Lagos, Lagos, Nigeria \\ ${ }^{4}$ Arrive Alive Diagnostics and Imaging Services, Lagos, Nigeria
}

Correspondence to: Dr. Oluyemi Aderemi. Email: remioluyemi@yahoo.com

Received: 28 Oct 2020 Revised: 6 Feb 2021 Accepted: 22 Mar 2021 Available online: 22 Apr 2021

\begin{abstract}
Summary
The term "microscopic colitis" (MC) is used to describe a chronic inflammatory bowel disease that includes two main subtypes based on histopathologic features: collagenous and lymphocytic. Scientific literature is replete with documentation of the disease from various regions of the world. However, the condition is rarely described in black African patients. We herein present the details of the clinical aspects and endoscopic findings of 3 Nigerian patients with the lymphocytic variant of MC. A short literature review of the clinical, endoscopic, and pathologic features of this rare
\end{abstract}

condition as well as other relevant aspects of $\mathrm{MC}$ is also presented.

Keywords: Microscopic colitis, Lymphocytic colitis, Nigeria.

Ann Afr Surg. 2021; 18(3): 180-184

DOI: http://dx.doi.org/10.4314/aas.v18i3.10

Conflicts of Interest: None

Funding: None

(C) 2021 Author. This work is licensed under the Creative Commons Attribution 4.0 International License.

\section{Introduction}

Microscopic colitis (MC) is a known cause of chronic, non-bloody diarrhea. The term itself was first introduced in 1980 by Read et al. (1). The classical description of $\mathrm{MC}$ as not having any visible anomaly on colonoscopic examination means that the diagnosis rests on specific histologic findings in colonic biopsies demonstrating either lymphocytic colitis (LC) or collagenous colitis (CC), which are the subtypes of MC (2). It is a disease that affects different nationalities and has varying prevalence rates across the continents (2). However, reports of this condition among black Africans are sparse (3). The reason for this in Nigeria might be related to our limited use of colonoscopy for evaluation of gastrointestinal (GI) disease and the lack of adequate histopathologic infrastructure and personnel for identification of uncommon GI conditions.

However, there has been an increase in the use of colonoscopy across the country. Therefore, as might be expected, there will be an increase in the number of such diagnoses. This article reviewed the database of a private out-patient endoscopy services center in Lagos and aimed to document the important aspects of the cases of histologically diagnosed MC, all of them of the LC sub-type.

\section{Case series}

Over the course of 38 months (from February 2016 to April 2019), 400 colonoscopies were carried out at a private endoscopy facility based in Lagos. The biopsy 
results from these procedures were reviewed, and only 3 cases were diagnosed as MC on histology (Fig. 1). In another instance, the suspicion of LC was raised. Despite an increase in the number of intraepithelial lymphocytes (IELs), this particular case was excluded from this case series because the histologic criterion of having >20 IELs per 100 epithelial cells was not met. The records were then reviewed for relevant clinical data and endoscopic findings in the identified cases.

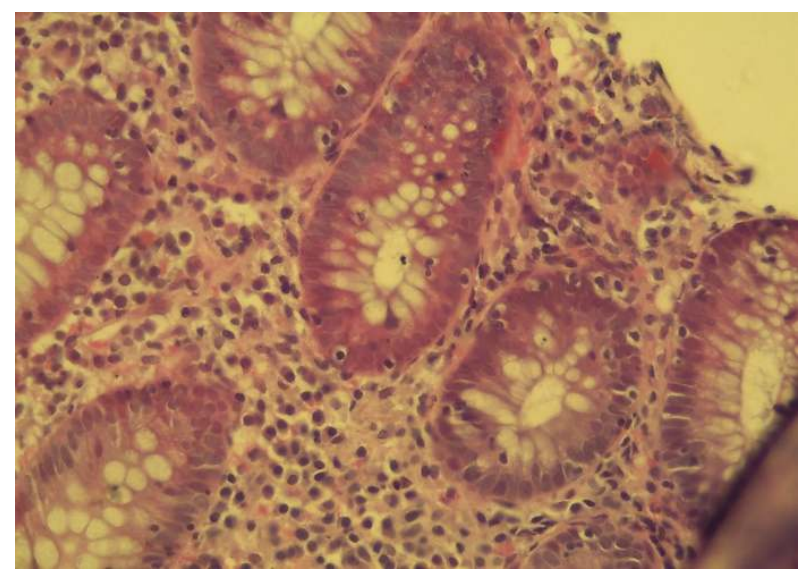

Figure 1. High-power photomicrograph of the rectal mucosa. The rectal mucosa has intraepithelial and lamina propria lymphocytic infiltrates ( $>20 / 100$ intraepithelial cells).

Several dilated capillaries are seen with few neutrophils and eosinophils. There is no evidence of subepithelial collagen bands (hematoxylin and eosin, original magnification $\times 400$ ).

\section{Case 1}

A 70-year-old man presented with a 6-month history of passage of watery stools. The rectal wall was markedly edematous and friable, and a longitudinal ulcer was also noted (Fig. 2). The rest of the mucosa lining was observed to be erythematous. Samples from the rectum and random biopsies of the other inflamed parts of the left side were reported as LC.

\section{Case 2}

A 66-year-old man was referred for his yearly colonoscopy approximately 4 months earlier than his scheduled checkup because of diarrhea. He undergoes annual colonoscopy because part of his colon was surgically removed 2 years prior as treatment for transverse colon cancer. He had no history of radiotherapy. The mucosal lining in the cecum was edematous with associated exuberance of the colonic folds. A striking attenuation to complete absence of the usual vascular markings was observed as well (Fig. 3). Biopsy reports for the cecal samples indicated LC.

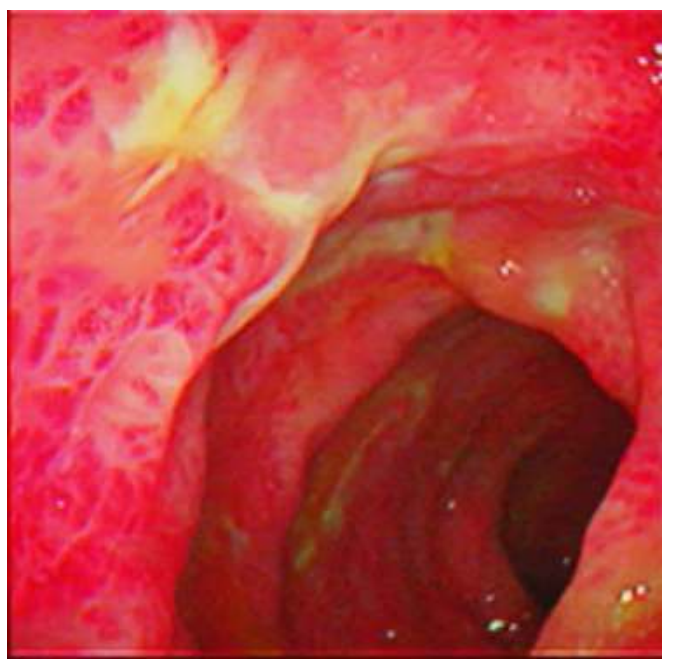

Figure 2. Endoscopic image of the rectum of case 1. The rectum had a markedly edematous and friable wall and a longitudinal ulcer.

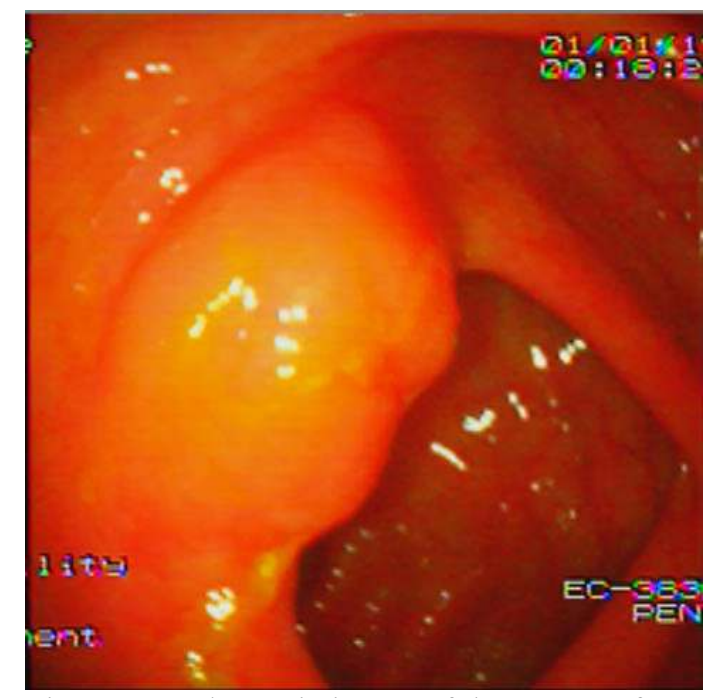

Figure 3. Endoscopic image of the cecum of case 2. The cecum had diffuse edema with exuberance of the colonic folds as well as striking attenuation to complete absence of the usual vascular markings.

\section{Case 3}

A 50-year-old woman presented with a 4-month history of diarrhea. Long before these bouts of watery stools, she had noticed intermittent drops of blood whenever she strained to pass stools. During colonoscopy, second- 
degree hemorrhoids with evidence of recent bleeding were observed. The patient's rectum and rectosigmoid regions were edematous and intensely erythematous as well (Fig. 4); the pattern of exudative hemorrhagic changes in this friable mucosa was reminiscent of barotrauma. The more proximal aspects of the left side of her colon were less intensely inflamed. Histopathology results of the random rectosigmoid biopsies indicated LC.

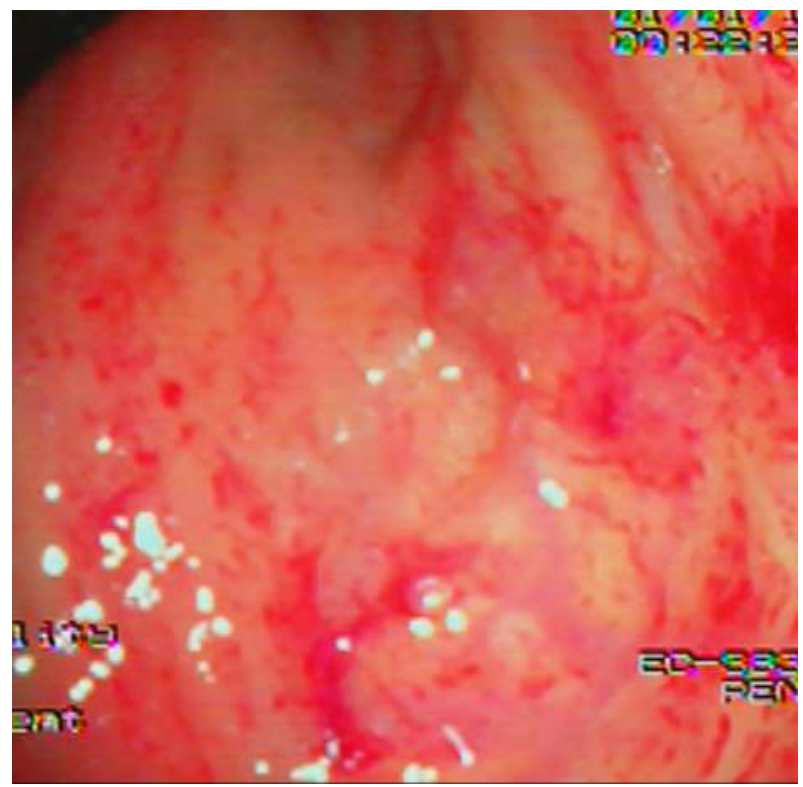

Figure 4. Endoscopic image of the rectum and rectosigmoid regions of case 3 . The rectum and rectosigmoid regions were edematous and erythematous. This pattern of "exudative hemorrhagic" changes was reminiscent of barotrauma.

\section{Discussion}

Although the worldwide prevalence of MC has been investigated, African data on the topic have been sparse $(2,3)$. This case series documented a few cases in Nigeria and reviewed some important aspects of their presentation in relation to the emerging concepts in $\mathrm{MC}$ management.

The typical clinical presentation of MC is chronic, nonbloody diarrhea usually in women in their mid-60s or older. Only two cases of the disease have been documented in Nigeria, one in a 65-year-old man and another in a 53-year-old woman; these cases had classic histories of chronic diarrhea but with normal endoscopic findings $(4,5)$.
Our patients were in their fifth decade of life or older, and two of them were men. Chronic diarrhea was the primary complaint in all our patients as was the case in the first reports from Nigeria. The third case, however, had accompanying intermittent hematochezia due to hemorrhoids.

The classical definition of MC as a diagnosis based on the absence of colonoscopy findings has been variously challenged, first in CC and then in LC (6-8). It has been resolved that the histologic criteria-based diagnosis is favored over this somewhat dated definition, as macroscopic endoscopic findings in $\mathrm{MC}$ have been well documented $(2,9)$. Endoscopic changes are seen in as much as $15 \%$ of cases (10). This important shift in the description of the disease is of vital importance in the management of $\mathrm{MC}$ in our environment. This is because we have limited facilities for endoscopy, detailed biopsy, and histopathological identification that this new shift in diagnosis will require. The additional cost of these often extensive multiple biopsies is a firm deterrent for many of our patients who pay out of pocket for medical care.

Macroscopic mucosal changes in $\mathrm{MC}$ are more commonly seen in instances of $\mathrm{CC}$ and not so much in the cases of LC. An exhaustive review of these changes, as presented by Koulaouzidis and Saeed (7), could only point to one such documentation in LC patients. Since then, there has been a review of LC changes in a Korean cohort of patients (8). In the said publication, hypervascularity, "exudative bleeding" (described as hemorrhage or oozing of blood from mucosal leaks and tending to cluster together in affected regions), and loss of normal vascularity were observed in half of all LC patients.

The Korean findings were illustrated by patients in this case series. Case 3 had exudative bleeding changes, which, on endoscopy, appeared to be reminiscent of barotrauma. The cecum in case 2 was edematous with attenuation and near loss of the usual vascular markings. The findings in case 1, however, appeared distinct, as intense, diffuse erythema in the rectum along with deep, linear ulcer-type defects has not been described in LC (only in CC) $(8,11)$. 
The use of 20 IELs per 100 epithelial cells has been long considered as the definitive criterion for LC diagnosis $(2,12)$. The added benefit of immunohistochemistry using CD3 in identifying and quantifying IELs has been shown in a South African report (13). However, the landscape of MC's histopathological definition has been challenged, in recent times, to include such entities as incomplete MC (14), MC not otherwise specified (15), and paucicellular LC $(16,17)$.

In these cases, the patients have the same clinical features of MC but fall short of the histologic criteria for diagnosis. We emphasize this because the histology of one of the patients who presented with diarrhea showed increased IELs as well, but the IEL count did not reach the required number (20 IELs) to make a definitive diagnosis; thus, he was excluded from this series. Perhaps, the case qualifies for the "incomplete MC" or the "paucicellular LC" category.

The implications of the colonic topographic distribution of $\mathrm{MC}$ changes on its diagnosis is a controversial issue. A rather dated report opined that full-length biopsies are essential for diagnosis, particularly of the LC sub-type (18). However, a more recent prospective, multicenter study from France revealed that biopsies from a short colonoscopy to the transverse colon were enough to clinch the diagnosis in more than $90 \%$ of cases (19). The latter stance was supported by Moore et al. (10) in a study in 2018. This point is important in Africa, as sigmoidoscopies are much more readily available, accessible, and affordable. Additionally, the viability and cost implications of random, full-length biopsy reports must be considered. For instance, all colonoscopic biopsies in the center where the study was carried out are taken from areas where there were macroscopic abnormalities.

The prognosis of the condition appears encouraging, as clinical remission is the rule. The deployment of budesonide and other therapeutic options has meant that a high proportion of patients (more than two thirds) enter clinical remission with care (10). The benign course of the disease is also well documented by other studies as well (20), and measures to be taken must be carefully weighed against their risks.
The work presented here documents the rare finding of $\mathrm{MC}$ in Nigerian patients along with some important aspects of their clinical and endoscopic presentations. A limitation of the retrospective nature of this study is that it did not allow us to probe into the full background (social, medical, and drug histories) of the patients or the treatment options and outcomes in each case.

\section{References}

1. Read NW, Krejs GJ, Read MG, et al. Chronic diarrhea of unknown origin. Gastroenterology 1980; 78: 264-271.

2. Münch A, Aust D, Bohr J, et al. Microscopic colitis: current status, present and future challenges: statements of the European Microscopic Colitis Group. European Microscopic Colitis Group (EMCG). J Crohns Colitis 2012; 6(9): 932-945.

3. Otegbayo JA, Otegbeye FM, Rotimi O. Microscopic colitis syndrome - a review article. J Natl Med Assoc 2005; 97(5): 678-682.

4. Ekrikpo UE, Otegbayo JA, Oluwasola AO. Lymphocytic colitis presenting as difficult diarrhoea in an African woman: a case report and review of the literature. J Med Case Rep 2010; 4: 31.

5. Otegbayo JA, Oluwasola AO, Akang EE. Collagenous colitis in an adult patient with chronic diarrhoea: case report. East Afr Med J 2001; 78(5): 272-274.

6. Cimmino DG, Mella JM. Endoscopic manifestations and mucosal patterns associated to collagenous colitis. In: Miskovitz P, ed. Colonoscopy. InTech; 2011. Available from:

https:/www.intechopen.com/books/colonoscopy/endosco pic-manifestations-and-mucosal-patterns-associated-tocollagenous-colitis.

7. Koulaouzidis A, Saeed AA. Distinct colonoscopic findings of microscopic colitis: not so microscopic after all? World J Gastroenterol 2011; 17(37): 4157-4165.

8. Park HS, Han DS, Ro YO, et al. Does lymphocytic colitis always present with normal endoscopic findings? Gut Liver 2015; 9(2): 197-201.

9. Fernández-Bañares F, Casanova MJ, et al. Current concepts on microscopic colitis: evidence-based statements and recommendations of the Spanish Microscopic Colitis Group. Aliment Pharmacol Ther 2016; 43(3): 400-26.

10. Moore M, Coleman HG, Allen PB, et al. Microscopic colitis: a population-based case series over a 9-year period in Northern Ireland. Colorectal Dis 2018; 20(11): 1020-1027.

11. Cuoco L, Bertoncello V, Salvagnini M. Colonic perforation after colonoscopy in patients with collagenous colitis. Am J Gastroenterol 2009; 104: 1846-1847. 
12. Magro F, Langner C, Driessen A, et al., for the European Society of Pathology. European consensus on the histopathology of inflammatory bowel disease. J Crohns Colitis 2013; 7(10): 827-852.

13. Mohamed N, Marais M, Bezuidenhout J. Microscopic colitis as a missed cause of chronic diarrhea. World $\mathrm{J}$ Gastroenterol 2011; 17(15): 1996-2002.

14. Bjørnbak C, Engel PJ, Nielsen PL, et al. Microscopic colitis: clinical findings, topography and persistence of histopathological subgroups. Aliment Pharmacol Ther 2011; 34(10): 1225-1234.

15. Warren BF, Edwards CM, Travis SP. 'Microscopic colitis': classification and terminology. Histopathology 2002; 40(4): 374-376.

16. Goldstein NS, Bhanot P. Paucicellular and asymptomatic lymphocytic colitis: expanding the clinicopathologic spectrum of lymphocytic colitis. Am J Clin Pathol 2004; 122(3): 405-411.

17. Fernández-Bañares $\mathrm{F}$, Casalots $\mathrm{J}$, Salas $\mathrm{A}$, et al. Paucicellular lymphocytic colitis: is it a minor form of lymphocytic colitis? A clinical pathological and immunological study. Am J Gastroenterol 2009; 104(5): 1189-1198.

18. Thijs WH, van Baarlen J, Kleibeuker JH, Kolkman JH. Microscopic colitis: prevalence and distribution throughout the colon in patients with chronic diarrhoea. Neth J Med 2005; 63(4): 137-140.

19. Macaigne G, Lahmek P, Locher C, et al., for the COLMI Group of the Association Nationale des Gastroentérologues des Hôpitaux (ANGH). Over 90\% of cases of microscopic colitis can be diagnosed by performing a short colonoscopy. Clin Res Hepatol Gastroenterol 2017; 41(3): 333-340.

20. Mullhaupt B, Güller U, Anabitarte M, et al. Lymphocytic colitis: clinical presentation and long-term course. Gut 1998; 43: 629-633. 